\title{
ON MILMAN'S MODULI FOR BANACH SPACES
}

\author{
ELISABETTA MALUTA, STANISŁAW PRUS, \\ AND MARIUSZ SZCZEPANIK
}

Received 3 November 2000

We show that infinite dimensional geometric moduli introduced by Milman are strongly related to nearly uniform convexity and nearly uniform smoothness. An application of those moduli to fixed point theory is given.

\section{Introduction}

Classical moduli of convexity and smoothness of Banach spaces were introduced in $[5,14]$, respectively. They describe the shape of two-dimensional sections of the unit ball of a space. Many applications of those moduli concern, however, problems which are not of two-dimensional character. This was the reason for introducing moduli which reflect multi-dimensional geometric structure of the unit ball.

In [16] (see also [18]), Milman developed $\beta$ and $\delta$ averaging procedures for scalar functions on the unit sphere with respect to a family of subspaces of a Banach space. He used those procedures to define various geometric moduli. Some of them describe properties which may be seen as counterparts of uniform convexity. Indeed, Figiel [7] established a formula relating the classical modulus of convexity to one of Milman's moduli. The idea used by Milman was different from the classical one, so many authors tried to find more direct generalizations of uniform convexity. In [22], Sullivan introduced $k$-dimensional uniform convexity and in $[10,11]$ infinite dimensional uniform convexity was defined. It turned out however that $k$-uniform convexity of Sullivan can be characterized in terms of $\delta$-moduli of Milman (see [13]). The dual property is in turn strongly related to $\beta$-moduli (see $[2,15]$ ).

In this paper, we study infinite dimensional Milman's moduli. In their definitions, subspaces with finite codimension are used. We establish new formulae for these moduli in Banach spaces with shrinking $M$-bases. In our formulae 
weakly null sequences are used. This enables us to show that in the infinite dimensional case $\beta$-moduli correspond to nearly uniform convexity introduced in [11] and $\delta$-moduli correspond to the dual property which was characterized in [19]. In particular we obtain a counterpart of Figiel's formula. We also give an application to the fixed point theory. Namely, we show that the weakly convergent sequence coefficient studied in this theory can be estimated in terms of $\beta$-moduli.

\section{Results}

In this paper, we consider only infinite dimensional Banach spaces. Let $X$ be such a space. By $B_{X}$ and $S_{X}$ we denote its closed unit ball and the unit sphere, respectively. The moduli studied in this paper are obtained via the so-called averaging procedures. The first two of them were considered in [16]. Let $\mathscr{B}$ be the family of all closed subspaces of $X$ with finite codimension. Given a function $f: X \rightarrow \mathbb{R}$, we define its $\beta$ and $\delta$ averaging as follows

$$
\beta f=\sup _{E \in \mathscr{B}} \inf _{y \in S_{E}} f(y), \quad \delta f=\inf _{E \in \mathscr{B}} \sup _{y \in S_{E}} f(y) .
$$

In this paper, we also use other averaging procedures. A Banach space $X$ is said to have the Schur property if every weakly convergent sequence in $X$ is norm convergent. It is well known that all finite dimensional spaces and the space $l_{1}$ have this property. On the other hand, if an infinite dimensional space does not contain an isomorphic copy of $l_{1}$, then it does not have the Schur property. Given a space $X$ without the Schur property, let $\mathcal{N}$ denote the set of all weakly null sequences $\left(x_{n}\right)$ in $S_{X}$, and let $f$ be a real-valued function on $X$. We put

$$
b f=\sup _{\left(y_{n}\right) \in \mathcal{N}} \inf _{n \in \mathbb{N}} f\left(y_{n}\right), \quad d f=\inf _{\left(y_{n}\right) \in \mathcal{N}} \sup _{n \in \mathbb{N}} f\left(y_{n}\right) .
$$

Now fix $\epsilon \geq 0$ and $x \in X$. We apply the averaging procedures to the function $f$ given by the formula

$$
f(y)=\|x+\epsilon y\|-1
$$

where $y \in X$. This gives the following quantities

$$
\beta(\epsilon ; x)=\beta f, \quad \delta(\epsilon ; x)=\delta f, \quad b(\epsilon ; x)=b f, \quad d(\epsilon ; x)=d f .
$$

Considering them as functions of $x \in X$, we set

$$
\begin{aligned}
& \beta \beta(\epsilon)=\beta \beta(\epsilon ; x), \\
& b b(\epsilon)=b b(\epsilon ; x), \quad d d(\epsilon)=\delta \delta(\epsilon ; x), \\
& b d(\epsilon ; x) .
\end{aligned}
$$


Additionally, we write

$$
\begin{array}{ll}
\tilde{\beta}(\epsilon)=\inf _{x \in S_{X}} \beta(\epsilon ; x), & \tilde{\delta}(\epsilon)=\sup _{x \in S_{X}} \delta(\epsilon ; x), \\
\tilde{b}(\epsilon)=\sup _{x \in S_{X}} b(\epsilon ; x), & \tilde{d}(\epsilon)=\inf _{x \in S_{X}} d(\epsilon ; x) .
\end{array}
$$

The functions of variable $\epsilon \geq 0$ defined by (2.5) and (2.6) will be called moduli of the space $X$. To avoid confusion in some cases we will add the name of a space as a subscript to the symbol of a modulus. The moduli obtained by $\beta$ and $\delta$ averaging procedures were intensively studied by Milman (see $[16,17]$ ). Functions strictly related to $\tilde{b}$ and $\tilde{d}$ can be found, for instance, in [8].

LEMMA 2.1. Let $\phi$ be one of the moduli defined in (2.5) or (2.6). Then

(i) $\phi(0)=0$ and $\phi$ is nonnegative,

(ii) $\phi$ satisfies the Lipschitz condition with the constant 1 ,

(iii) the function $\phi(\epsilon) / \epsilon$ is nondecreasing on $(0, \infty)$.

Proof. Fix $\epsilon>0$ and $x \in S_{X}$. We show that in this case the quantities defined by (2.4) are nonnegative. For this purpose, we choose a functional $x^{*} \in S_{X^{*}}$ so that $x^{*}(x)=1$. Then

$$
\|x+\epsilon y\| \geq x^{*}(x+\epsilon y)=1
$$

for every $y \in \operatorname{ker} x^{*}$. It follows that

$$
\inf _{y \in S_{E}}\|x+\epsilon y\|-1 \geq 0
$$

where $E=\operatorname{ker} x^{*}$ and, consequently, $\beta(\epsilon ; x) \geq 0$. One can similarly show that $\delta(\epsilon ; x) \geq 0$. If $X$ does not have the Schur property, then the same is true for $\operatorname{ker} x^{*}$. The space $\operatorname{ker} x^{*}$ contains therefore a sequence $\left(y_{n}\right) \in \mathcal{N}$ and from (2.7) we see that $b(\epsilon ; x) \geq 0$. The inequality $d(\epsilon ; x) \geq 0$ follows in turn from the estimate

$$
\sup _{n \in \mathbb{N}}\left\|x+\epsilon y_{n}\right\| \geq \liminf _{n \rightarrow \infty}\left\|x+\epsilon y_{n}\right\| \geq\|x\|=1
$$

for every $\left(y_{n}\right) \in \mathcal{N}$.

Property (ii) is a direct consequence of the triangle inequality. To prove (iii) it is enough to observe that for each $x, y \in S_{X}$ the function $\psi(\epsilon)=\|x+\epsilon y\|$ is convex on $(0, \infty)$. Its divided difference

$$
\frac{1}{\epsilon}(\psi(\epsilon)-\psi(0))=\frac{1}{\epsilon}(\|x+\epsilon y\|-1)
$$

is therefore a nondecreasing function of $\epsilon$. 
Lemma 2.2. Let $X$ be a Banach space without the Schur property. If $\epsilon \geq 0$ and $x \in X$, then

$$
d(\epsilon ; x)=\inf \sup _{n \in \mathbb{N}}\left\|x+\epsilon y_{n}\right\|-1,
$$

where the infimum is taken over all weakly null sequences $\left(y_{n}\right)$ in $X$ such that $\left\|y_{n}\right\| \geq 1$ for every $n$. Moreover,

$$
\tilde{d}(\epsilon)=\inf \{d(\epsilon ; x): x \in X,\|x\| \geq 1\},
$$

for every $\epsilon \in[0,1]$.

Proof. Fix $\epsilon \geq 0, x \in X$ and a weakly null sequence $\left(y_{n}\right)$ in $X$ such that $\left\|y_{n}\right\| \geq 1$ for every $n$. Since for each $n$ the function $t \mapsto\left\|x+\epsilon t y_{n}\right\|$ is convex,

$$
\left\|x+\epsilon y_{n}^{\prime}\right\|-\|x\| \leq \frac{1}{\left\|y_{n}\right\|}\left(\left\|x+\epsilon y_{n}\right\|-\|x\|\right) \leq \frac{1}{\left\|y_{n}\right\|}\left(\sup _{m \in \mathbb{N}}\left\|x+\epsilon y_{m}\right\|-\|x\|\right),
$$

where $y_{n}^{\prime}=\left(1 /\left\|y_{n}\right\|\right) y_{n}$. But $\sup _{m \in \mathbb{N}}\left\|x+\epsilon y_{m}\right\| \geq\|x\|$. We therefore see that

$$
\sup _{n \in \mathbb{N}}\left\|x+\epsilon y_{n}^{\prime}\right\| \leq \sup _{n \in \mathbb{N}}\left\|x+\epsilon y_{n}\right\| .
$$

This gives formula (2.11).

Now assume that $\|x\| \geq 1,\left(y_{n}\right) \in \mathcal{N}$ and $\epsilon \in[0,1]$. We have

$$
\|x\|\left(\left\|x^{\prime}+\epsilon y_{n}\right\|-\epsilon\right) \leq\left\|x+\epsilon y_{n}\right\|-\epsilon,
$$

for every $n$ where $x^{\prime}=(1 /\|x\|) x$. It follows that

$$
\|x\|\left(d\left(\epsilon ; x^{\prime}\right)-\epsilon+1\right) \leq d(\epsilon ; x)-\epsilon+1 .
$$

But the proof of Lemma 2.1 shows that $d\left(\epsilon ; x^{\prime}\right) \geq 0 \geq \epsilon-1$. From (2.11) and (2.16) we therefore obtain

$$
\tilde{d}(\epsilon) \leq d\left(\epsilon ; x^{\prime}\right) \leq d(\epsilon ; x) .
$$

This easily implies (2.12).

Remark 2.3. A reasoning similar to that in the proof of Lemma 2.2 shows that in each of the formulae (2.4), (2.5), and (2.6) the unit sphere can be replaced by the set of all elements outside of the open unit ball if it occurs with infimum and by the unit ball if it occurs with supremum. Moreover, one can replace $\mathcal{N}$ by the set of all weakly null sequences outside of the open unit ball when dealing with infimum and by the set of all weakly null sequences in the unit ball when dealing with supremum. In case of formulae (2.5) and (2.6) this requires the assumption that $\epsilon \in[0,1]$. 
The results in the above remark are analogous to those proved for the classical moduli of convexity and smoothness in [5, 14].

Let $X$ be a space without the Schur property. Given $\epsilon \in[0,1]$, we put

$$
K(\epsilon)=\inf \{1-\|x\|\},
$$

where the infimum is taken over all weak limits $x$ of sequences $\left(x_{n}\right)$ in $B_{X}$ such that $\left\|x_{n}-x\right\| \geq \epsilon$ for all $n$. An infinite dimensional Banach space $X$ is nearly uniformly convex (NUC for short) if $X$ is reflexive and $K(\epsilon)>0$ for every $\epsilon>0$ (see [11]). The property dual to NUC was studied in [19]. It is called nearly uniform smoothness (NUS for short).

Lemma 2.4. Let $X$ be a Banach space without the Schur property. Then $K$ is continuous on the interval $[0,1)$ and the function $K(\epsilon) / \epsilon$ is nondecreasing on $(0,1)$.

Proof. We follow an idea from [9]. Fix $u \in S_{X}$ and $\left(u_{n}\right) \in \mathcal{N}$. Having $\epsilon \in[0,1]$, by $K\left(\epsilon ; u,\left(u_{n}\right)\right)$ we denote the infimum of all numbers $t \in[0,1]$ for which there is a scalar sequence $\left(s_{n}\right)$ such that $\left\|(1-t) u+s_{n} u_{n}\right\| \leq 1$ and $s_{n} \geq \epsilon$ for all $n$.

It is easy to check that $K\left(\epsilon ; u,\left(u_{n}\right)\right)$ is a convex function of $\epsilon$ and

$$
K(\epsilon)=\inf \left\{K\left(\epsilon ; u,\left(u_{n}\right)\right): u \in S_{X},\left(u_{n}\right) \in \mathcal{N}\right\},
$$

for every $\epsilon \in[0,1]$. Consequently, for each $s \in[0,1)$ the function $K$ satisfies the Lipschitz condition with the constant $1 /(1-s)$ on the interval $[0, s)$. Moreover, $K\left(0 ; u,\left(u_{n}\right)\right)=0$ for all $u \in S_{X}$ and $\left(u_{n}\right) \in \mathcal{N}$ which gives the second part of the conclusion.

Now recall the definition of a shrinking $M$-basis. A family $\left\{e_{t}\right\}_{t \in T}$ of vectors of a Banach space $X$ is called an $M$-basis of $X$ if there exist functionals $e_{t}^{*} \in X^{*}$, $t \in T$ such that

$$
e_{t}^{*}\left(e_{s}\right)= \begin{cases}0 & \text { if } t \in T \backslash\{s\}, \\ 1 & \text { if } t=s,\end{cases}
$$

and $\left\{e_{t}\right\}_{t \in T}$ is linearly dense in $X$. If in addition $\left\{e_{t}^{*}\right\}_{t \in T}$ is linearly dense in $X^{*}$, then $\left\{e_{t}\right\}_{t \in T}$ is said to be shrinking. Given $x \in \operatorname{span}\left\{e_{t}\right\}_{t \in T}$, we put supp $x=\{t \in$ $\left.T: e_{t}^{*}(x) \neq 0\right\}$. We point out that all reflexive spaces have shrinking $M$-bases (see [21, page 716]).

THEOREM 2.5. Let $X$ be a Banach space without the Schur property and let $f, g: X \rightarrow \mathbb{R}$ be functions such that $f$ is uniformly continuous and $f(x) \leq g(x)$ for every $x \in S_{X}$. Then $\beta f \leq d g$ and $b f \leq \delta g$. Moreover, if $X$ is a subspace of a Banach space with a shrinking $M$-basis, then $\beta f=d f$ and $\delta f=b f$.

Proof. We fix $\gamma>0$. By our assumption there is $\eta>0$ such that if $x, y \in X$, $\|x-y\| \leq \eta$, then $|f(x)-f(y)| \leq \gamma$. We choose $E \in \mathscr{B}$ and $\left(y_{n}\right) \in \mathcal{N}$ so 
that $\beta f-\gamma \leq \inf _{y \in S_{E}} f(y)$ and $\sup _{n \in \mathbb{N}} g\left(y_{n}\right) \leq d g+\gamma$. Put $m=\operatorname{codim} E$. There are elements $x_{1}, \ldots, x_{m} \in S_{X}$ and functionals $x_{1}^{*}, \ldots, x_{m}^{*} \in X^{*}$ such that $E=\bigcap_{i=1}^{m} \operatorname{ker} x_{i}^{*}, x_{k}^{*}\left(x_{k}\right) \neq 0$ and $x_{k}^{*}\left(x_{i}\right)=0$ for every $i=1, \ldots, m$ and $k \neq i$.

We set $\eta_{1}=\eta\left(2 \sum_{i=1}^{m} 1 /\left|x_{i}^{*}\left(x_{i}\right)\right|\right)^{-1}$ and find $s$ for which $\left|x_{k}^{*}\left(y_{s}\right)\right|<\eta_{1}$, $k=1, \ldots, m$. Consider an element

$$
y^{\prime}=y_{s}-\sum_{i=1}^{m} x_{i}^{*}\left(y_{s}\right)\left(x_{i}^{*}\left(x_{i}\right)\right)^{-1} x_{i} .
$$

Clearly, $x_{k}^{*}\left(y^{\prime}\right)=0$ for $k=1, \ldots, m$ which shows that $y^{\prime} \in E$. Moreover, $\| y_{s}-$ $y^{\prime} \|<\eta / 2$ and consequently $\left\|y_{s}-y\right\|<\eta$ where $y=\left(1 /\left\|y^{\prime}\right\|\right) y^{\prime}$. Hence $f(y)-$ $f\left(y_{s}\right) \leq \gamma$, so

$$
\beta f-\gamma \leq f(y) \leq g\left(y_{s}\right)+\gamma \leq d g+2 \gamma .
$$

Since $\gamma>0$ is arbitrary, this gives the inequality $\beta f \leq d g$. The inequality $b f \leq \delta g$ can be proved in a similar way.

Now assume that $\beta f \neq d f$. From the first part of the proof it follows that we can find $c \in \mathbb{R}$ so that $\beta f<c<d f$. Consequently, for every $E \in \mathscr{B}$ there is $y \in S_{E}$ such that $f(y)<c$.

Let $Z$ be the space with the shrinking $M$-basis $\left(\left\{x_{t}\right\}_{t \in T},\left\{x_{t}^{*}\right\}_{t \in T}\right)$ which contains $X$ as a subspace. We put $Y=\operatorname{span}\left\{x_{t}\right\}_{t \in T}$ and fix $\gamma>0$. By induction we choose two sequences; $\left(y_{n}\right)$ in $S_{X}$ and $\left(u_{n}\right)$ in $Y$ so that $f\left(y_{n}\right)<c, \| y_{n}-$ $u_{n} \|<\gamma / n$ for every $n$ and $\operatorname{supp} u_{i} \cap \operatorname{supp} u_{j}=\emptyset$ if $i \neq j$.

By assumption, there is $y_{1} \in S_{X}$ such that $f\left(y_{1}\right)<c$ and we find $u_{1} \in Y$ with $\left\|y_{1}-u_{1}\right\|<\gamma$. Next, having $y_{1}, \ldots, y_{n-1}$ and $u_{1}, \ldots, u_{n-1}$, we set $T_{1}=$ $\bigcup_{i=1}^{n-1} \operatorname{supp} u_{i}, E_{1}=\bigcap_{t \in T_{1}} \operatorname{ker} x_{t}^{*}$. By assumption, there exists $y_{n} \in S_{E_{1}} \cap X$ such that $f\left(y_{n}\right)<c$. We choose $u \in Y$ for which $\left\|y_{n}-u\right\|<\gamma_{1}$ where $\gamma_{1}=$ $\gamma\left(2 n \max \left\{1, \sum_{t \in T_{1}}\left\|x_{t}\right\|\left\|x_{t}^{*}\right\|\right\}\right)^{-1}$ and set

$$
u_{n}=u-\sum_{t \in T_{1}} x_{t}^{*}(u) x_{t} .
$$

Clearly, $\operatorname{supp} u_{n} \cap T_{1}=\emptyset$ and

$$
\left\|u_{n}-u\right\| \leq \sum_{t \in T_{1}}\left|x_{t}^{*}\left(u-y_{n}\right)\right|\left\|x_{t}\right\| \leq \sum_{t \in T_{1}}\left\|x_{t}^{*}\right\|\left\|x_{t}\right\|\left\|u-y_{n}\right\|<\frac{\gamma}{2 n} .
$$

Therefore $\left\|y_{n}-u_{n}\right\|<\gamma / n$.

Since the $M$-basis of $Z$ is shrinking, $\left(u_{n}\right)$ is weakly null and consequently $\left(y_{n}\right) \in \mathcal{N}$. Hence $d f \leq \sup _{n \in \mathbb{N}} f\left(y_{n}\right) \leq c$ which is a contradiction. The formula $\delta f=b f$ can be shown in a similar way.

Corollary 2.6. Let $X$ be a Banach space without the Schur property and $x \in$ $S_{X}$. Then

$$
\begin{array}{cc}
\beta(\epsilon ; x) \leq d(\epsilon ; x), & b(\epsilon ; x) \leq \delta(\epsilon ; x), \\
\beta \beta(\epsilon) \leq d d(\epsilon), & b b(\epsilon) \leq \delta \delta(\epsilon),
\end{array}
$$


for all $\epsilon \geq 0$. If $X$ is a subspace of a Banach space with a shrinking M-basis, then the equalities hold in each of these inequalities.

In what follows, we will show that some of the considered moduli are related to NUC. Dealing with such a modulus $\phi$ of a space $X$, we say that $X$ is $\phi$ uniformly convex if $\phi(\epsilon)>0$ for every $\epsilon>0$. Other moduli correspond to NUS and in this case we say that $X$ is $\phi$-uniformly smooth if the modulus $\phi$ of $X$ satisfies the condition $\lim _{\epsilon \rightarrow 0} \phi(\epsilon) / \epsilon=0$. For instance a reflexive space $X$ is NUS if and only if $X$ is $\tilde{b}$-uniformly smooth (see [19]). Our next result shows in turn that the moduli $K$ and $\tilde{d}$ are, in a sense, equivalent.

THEOREm 2.7. If a Banach space $X$ does not have the Schur property, then

$$
\begin{aligned}
& K\left(\frac{\epsilon}{1+\tilde{d}(\epsilon)}\right)=\frac{\tilde{d}(\epsilon)}{1+\tilde{d}(\epsilon)}, \\
& \tilde{d}\left(\frac{\epsilon}{1-K(\epsilon)}\right)=\frac{K(\epsilon)}{1-K(\epsilon)},
\end{aligned}
$$

for every $\epsilon \in[0,1)$.

Proof. Fix $\epsilon, \gamma \in(0,1)$. There are $x \in S_{X}$ and $\left(y_{n}\right) \in \mathcal{N}$ such that $\sup _{n \in \mathbb{N}} \| x+$ $\epsilon y_{n} \|-1<\tilde{d}(\epsilon)+\gamma$.

We put $u=(1+\tilde{b}(\epsilon)+\gamma)^{-1} x, u_{n}=\epsilon(1+\tilde{b}(\epsilon)+\gamma)^{-1} y_{n}$ and $z_{n}=u+u_{n}$ for $n \in \mathbb{N}$. Then $\left(z_{n}\right)$ is a sequence in $B_{X}$ converging weakly to $u$ and $\left\|z_{n}-u\right\|=$ $(1+\tilde{d}(\epsilon)+\gamma)^{-1}$ for every $n$. Therefore

$$
K\left(\frac{\epsilon}{1+\tilde{d}(\epsilon)+\gamma}\right) \leq 1-\|u\|=1-(1+\tilde{d}(\epsilon)+\gamma)^{-1} .
$$

In view of Lemma 2.4, we conclude that

$$
K\left(\frac{\epsilon}{1+\tilde{d}(\epsilon)}\right) \leq \frac{\tilde{d}(\epsilon)}{1+\tilde{d}(\epsilon)} .
$$

Lemma 2.4 shows also that if $0<\epsilon<1$, then $K(\epsilon)<1$. We assume now that $0<\gamma<1-K(\epsilon)$ and choose a sequence $\left(z_{n}\right)$ in $B_{X}$ so that $\left(z_{n}\right)$ converges weakly to $x,\left\|z_{n}-x\right\| \geq \epsilon$ for all $n$ and $1-\|x\|<K(\epsilon)+\gamma$. We put $v=$ $(1 /(1-K(\epsilon)-\gamma)) x, v_{n}=(1 / \epsilon)\left(z_{n}-x\right)$ for $n \in \mathbb{N}$. Then $\|v\|>1,\left\|v_{n}\right\| \geq 1$ for every $n$ and $\left(v_{n}\right)$ converges weakly to 0 . By Lemma 2.2,

$$
\tilde{d}\left(\frac{\epsilon}{1-K(\epsilon)-\gamma}\right) \leq \sup _{n \in \mathbb{N}}\left\|v+\frac{\epsilon}{1-K(\epsilon)-\gamma} v_{n}\right\|-1 \leq \frac{K(\epsilon)+\gamma}{1-K(\epsilon)-\gamma} .
$$

Using Lemma 2.1, we therefore get

$$
\tilde{d}\left(\frac{\epsilon}{1-K(\epsilon)}\right) \leq \frac{K(\epsilon)}{1-K(\epsilon)}
$$


To show the inequality opposite to (2.31) we set $\phi(t)=t /(1-K(t))$ for $t \in[0,1)$. From (2.29) we obtain

$$
\tilde{d}(\phi(\epsilon)) \geq(1+\tilde{d}(\phi(\epsilon))) K\left(\frac{\phi(\epsilon)}{1+\tilde{d}(\phi(\epsilon))}\right) .
$$

Moreover, (2.31) yields

$$
\frac{\phi(\epsilon)}{1+\tilde{d}(\phi(\epsilon))} \geq \phi(\epsilon)(1-K(\epsilon)) \geq \epsilon .
$$

This and Lemma 2.4 give

$$
\tilde{d}(\phi(\epsilon)) \geq \frac{K(\epsilon)}{\epsilon} \phi(\epsilon)=\frac{K(\epsilon)}{1-K(\epsilon)} .
$$

This is the inequality opposite to (2.31) and the proof of (2.27) is completed.

Now observe that $\phi$ is a continuous function on $[0,1)$ with $\phi(0)=0$ and $\lim _{\tau \rightarrow 1} \phi(\tau) \geq 1$. Having $\epsilon \in[0,1)$, we can therefore find $\tau \in[0,1)$ so that $\epsilon=\phi(\tau)$. Вy (2.27),

$$
\frac{\tilde{d}(\epsilon)}{1+\tilde{d}(\epsilon)}=\frac{\tilde{d}(\phi(\tau))}{1+\tilde{d}(\phi(\tau))}=K(\tau) .
$$

But $\epsilon(1-K(\tau))=\tau$, so

$$
\begin{aligned}
\frac{\tilde{d}(\epsilon)}{1+\tilde{d}(\epsilon)} & =K(\epsilon(1-K(\tau))) \\
& =K\left(\epsilon\left(1-\frac{\tilde{d}(\epsilon)}{1+\tilde{d}(\epsilon)}\right)\right) \\
& =K\left(\frac{\epsilon}{1+\tilde{d}(\epsilon)}\right) .
\end{aligned}
$$

Let $\delta_{X}$ be the classical modulus of convexity of a Banach space $X$ (cf. [6, page 124]). Given $\epsilon \geq 0$, we put

$$
\Delta_{X}(\epsilon)=\inf _{x, y \in S_{X}} \max \{\|x+\epsilon y\|,\|x-\epsilon y\|\}-1 .
$$

This modulus was also introduced in [16]. We have

$$
\Delta_{X}\left(\frac{\epsilon}{2\left(1-\delta_{X}(\epsilon)\right)}\right)=\frac{\delta_{X}(\epsilon)}{1-\delta_{X}(\epsilon)},
$$

for every $\epsilon \in[0,2)$ (see [7, Lemma 6] and [6, page 127]). Formula (2.27) can be seen as an infinite dimensional counterpart of (2.38). The reasoning used in the 
last part of the proof of Theorem 2.7 enables us to obtain the formula inverse to (2.38). Namely,

$$
\delta_{X}\left(\frac{2 \epsilon}{1+\Delta_{X}(\epsilon)}\right)=\frac{\Delta_{X}(\epsilon)}{1+\Delta_{X}(\epsilon)}
$$

for every $\epsilon \in[0,1)$.

From Theorem 2.7 and Corollary 2.6, we immediately get the next result.

COROLlary 2.8. If a Banach space $X$ is a subspace of a space with a shrinking M-basis, then

$$
K\left(\frac{\epsilon}{1+\tilde{\beta}(\epsilon)}\right)=\frac{\tilde{\beta}(\epsilon)}{1+\tilde{\beta}(\epsilon)}, \quad \tilde{\beta}\left(\frac{\epsilon}{1-K(\epsilon)}\right)=\frac{K(\epsilon)}{1-K(\epsilon)},
$$

for every $\epsilon \in[0,1)$.

This gives in turn the following result.

Corollary 2.9. Let $X$ be a reflexive space. Then $X$ is NUC if and only if $X$ is $\tilde{\beta}$-uniformly convex.

We show that formulae analogous to those in Theorem 2.7 hold for the modulus $\beta \beta$. In this case, we replace $K$ by the function $\bar{K}$ given by the formula

$$
\bar{K}(\epsilon)=\sup _{E \in \mathscr{B}} K_{E}(\epsilon)
$$

where $\epsilon \in[0,1]$.

THEOREM 2.10. Let a Banach space $X$ be a subspace of a space with a shrinking M-basis. Then

$$
\bar{K}\left(\frac{\epsilon}{1+\beta \beta(\epsilon)}\right)=\frac{\beta \beta(\epsilon)}{1+\beta \beta(\epsilon)}, \quad \beta \beta\left(\frac{\epsilon}{1-\bar{K}(\epsilon)}\right)=\frac{\bar{K}(\epsilon)}{1-\bar{K}(\epsilon)},
$$

for every $\epsilon \in[0,1)$.

Proof. Fix $\epsilon \in[0,1)$. From Corollary 2.6, we see that $\beta \beta(\epsilon)=\sup _{E \in \mathscr{B}} \tilde{d}_{E}(\epsilon)$ for every $\epsilon \geq 0$. This and Theorem 2.7 give the inequality

$$
K_{E}\left(\frac{\epsilon}{1+\beta \beta(\epsilon)}\right) \leq K_{E}\left(\frac{\epsilon}{1+\tilde{d}_{E}(\epsilon)}\right)=\frac{\tilde{d}_{E}(\epsilon)}{1+\tilde{d}_{E}(\epsilon)} \leq \frac{\beta \beta(\epsilon)}{1+\beta \beta(\epsilon)}
$$

for every $E \in \mathscr{B}$. It follows that

$$
\bar{K}\left(\frac{\epsilon}{1+\beta \beta(\epsilon)}\right) \leq \frac{\beta \beta(\epsilon)}{1+\beta \beta(\epsilon)}
$$


In order to show the opposite inequality, we take $\gamma>0$ and find $E \in \mathscr{B}$ so that $\beta \beta(\epsilon)-\gamma<\tilde{d}_{E}(\epsilon)$. By Theorem 2.7,

$$
\bar{K}\left(\frac{\epsilon}{1+\beta \beta(\epsilon)-\gamma}\right) \geq K_{E}\left(\frac{\epsilon}{1+\tilde{d}_{E}(\epsilon)}\right)=\frac{\tilde{d}_{E}(\epsilon)}{1+\tilde{d}_{E}(\epsilon)}>\frac{\beta \beta(\epsilon)-\gamma}{1+\beta \beta(\epsilon)} .
$$

The proof of Lemma 2.4 shows that $\bar{K}$ is continuous on $[0,1)$, so we can pass to the limit with $\gamma$ tending to 0 . This completes the proof of the first formula.

From Theorem 2.7, we also see that

$$
\beta \beta\left(\frac{\epsilon}{1-\bar{K}(\epsilon)}\right) \geq \tilde{d}_{E}\left(\frac{\epsilon}{1-K_{E}(\epsilon)}\right)=\frac{K_{E}(\epsilon)}{1-K_{E}(\epsilon)},
$$

for every $E \in \mathscr{B}$, and it shows that

$$
\beta \beta\left(\frac{\epsilon}{1-\bar{K}(\epsilon)}\right) \geq \frac{\bar{K}(\epsilon)}{1-\bar{K}(\epsilon)} .
$$

To prove the opposite inequality we take arbitrary $E, F \in \mathscr{B}$. Then $E \cap F \in \mathscr{B}$ and

$$
\tilde{d}_{E}\left(\frac{\epsilon}{1-K_{F}(\epsilon)}\right) \leq \tilde{d}_{E \cap F}\left(\frac{\epsilon}{1-K_{E \cap F}(\epsilon)}\right) \leq \frac{\bar{K}(\epsilon)}{1-\bar{K}(\epsilon)}
$$

It follows that

$$
\beta \beta\left(\frac{\epsilon}{1-\bar{K}(\epsilon)}\right) \leq \frac{\bar{K}(\epsilon)}{1-\bar{K}(\epsilon)}
$$

COROLlary 2.11. Let $X$ be a reflexive space. Then

(i) if $\beta \beta(1)>0$, then $X$ has an equivalent norm for which it is NUC,

(ii) if $\lim _{\epsilon \rightarrow 0} \delta \delta(\epsilon) / \epsilon<1$, then $X$ has an equivalent norm for which it is NUS.

Proof. Assume that $\beta \beta(1)>0$. By Theorem 2.10, there exists a subspace $E \in \mathscr{P}$ such that $\lim _{t \rightarrow 1} K_{E}(t)>0$. From [20, Theorem 2.11], we know that in this case $E$ has an equivalent norm in which it is NUC. Since $E$ has finite codimension, the same is true for the whole space $X$. In a similar way, one can derive (ii) from [20, Theorem 2.7].

Since NUS is dual to NUC (see [19, Theorem 2.4]), Corollary 2.11 shows that for reflexive spaces $\delta \delta$-uniform smoothness is isomorphically dual to $\beta \beta$ uniform convexity. In [17] (see also [16, page 118]), isometric duality of these properties was established for some class of spaces. Let $X$ be a Banach space and let $\mathscr{F}$ denote the family of all finite dimensional subspaces of $X$. Given 
$\epsilon \geq 0$, we put

$$
\bar{b}(\epsilon)=\inf _{Y \in \mathscr{F}} \tilde{b}_{X / Y}(\epsilon), \quad \bar{d}(\epsilon)=\sup _{Y \in \mathscr{F}^{F}} \tilde{d}_{X / Y}(\epsilon)
$$

A reasoning from the proof of Theorem 2.4 in [19] gives another duality result.

Proposition 2.12. Let $X$ be a reflexive space. Then

(i) $X$ is $\beta \beta$-uniformly convex if and only if $X^{*}$ is $\bar{b}$-uniformly smooth,

(ii) $X$ is $\delta \delta$-uniformly smooth if and only if $X^{*}$ is $\bar{d}$-uniformly convex.

We will give examples which show that in general $\beta \beta$-uniform convexity and $\delta \delta$-uniform smoothness are not dual to each other. Let $\|\cdot\|_{2}$ be the standard norm of $l_{2}$ and $\left(e_{n}\right)$ be the standard basis of this space. Given $x=\left(x_{n}\right) \in l_{2}$, we put $R x=\left(0, x_{2}, x_{3}, \ldots\right)$,

$$
\begin{aligned}
|x|_{0} & =\sup _{1<m<n}\left(\left|x_{m}-x_{1}\right|+\frac{1}{4}\left|x_{n}\right|\right), \\
\|x\|_{0} & =\max \left\{\|R x\|_{2},|x|_{0}\right\} .
\end{aligned}
$$

Clearly, $\|\cdot\|_{0}$ is a norm in $l_{2}$ equivalent to $\|\cdot\|_{2}$. Let $X_{0}$ be the space $l_{2}$ endowed with the norm $\|\cdot\|_{0}$. We have $\left\|e_{n}\right\|_{0}=1$ and $\left\|e_{m}+t e_{n}\right\|_{0}=1+(1 / 4) t$ for all $1<m<n$ and every $t \in[0,1 / 2]$. It follows that $b b(t) \geq(1 / 4) t$ for every $t \in[0,1 / 2]$. From Corollary 2.6, we therefore see that $X_{0}$ is not $\delta \delta$ uniformly smooth.

Let $Y$ be the subspace of $X_{1}$ spanned by $e_{1}$. We fix $x=\left(x_{n}\right)$ in $X_{0}$. Clearly, the norm $v$ of $x+Y$ in $X_{0} / Y$ does not exceed $\|R x\|_{2}$. To show the opposite inequality we consider two cases.

(I) $\left|x_{k}\right| \leq(4 / 5)\|R x\|_{2}$ for every $k \geq 2$. Then

$$
\left|x_{m}\right|+\frac{1}{4}\left|x_{n}\right| \leq\|R x\|_{2}
$$

where $1<m<n$.

(II) There is $k \geq 2$ for which $\left|x_{k}\right|>(4 / 5)\|R x\|_{2}$. Then $\left|x_{i}\right|<(3 / 5)\|R x\|_{2}$ whenever $i \geq 2, i \neq k$. We put $\lambda=\left(\operatorname{sign} x_{k}\right)(3 / 20)\|R x\|_{2}$. It is easy to see that

$$
\left|x_{m}-\lambda e_{1}\right|+\frac{1}{4}\left|x_{n}\right|<\|R x\|_{2},
$$

for all $1<m<n$.

It follows that $v \leq\|R x\|_{2}$ and finally $v=\|R x\|_{2}$. This shows that the space $X_{0} / Y$ is isometrically isomorphic to $l_{2}$. Consequently, $X_{0}$ is $\bar{b}$-uniformly smooth and by Proposition 2.12, $X_{0}^{*}$ is $\beta \beta$-uniformly convex. 
As the next example we consider the space $l_{2}$ endowed with an equivalent norm given by the formula

$$
\|x\|_{1}=\max \left\{\frac{4}{5}\|R x\|_{2},|x|_{1}\right\},
$$

where

$$
|x|_{1}=\sup _{1<m<n} \max \left\{\left|x_{m}-x_{1}\right|, \frac{4}{5}\left|x_{n}\right|\right\}
$$

for $x=\left(x_{k}\right) \in l_{2}$. We denote this space by $X_{1}$. Clearly, $\left\|e_{n}\right\|_{1}=1$ and $\| e_{m}+$ $t e_{n} \|_{0}=1$ for all $1<m<n$ and every $t \in[0,1 / 2]$. This shows that $d d(t)=0$ for every $t \in[0,1 / 2]$.

As in the preceding example, $X_{1} / Y$ is isometrically isomorphic to $l_{2}$. In fact, the norm $v$ of a coset $x+Y$ in $X_{1} / Y$ equals (4/5) $\|R x\|_{2}$. Indeed, the inequality $v \leq(4 / 5)\|R x\|_{2}$ is obvious. To show the opposite one, we can apply the same reasoning as before but this time in the case (II) we put $\alpha=$ $\left(\operatorname{sign} x_{k}\right)(1 / 5)\|R x\|_{2}$. Thus $X_{1}^{*}$ is $\delta \delta$-uniformly smooth while by Corollary 2.6, $X_{1}$ is not $\beta \beta$-uniformly convex.

NUS spaces have the Banach-Saks property (see [19, Corollary 3.4]). We will extend this result. First recall that a Banach space is said to have the weak Banach-Saks property if every weakly convergent sequence $\left(x_{n}\right)$ in $X$ has a subsequence $\left(x_{n_{k}}\right)$ such that the means $(1 / m) \sum_{k=1}^{m} x_{n_{k}}$ converge in norm.

Proposition 2.13. Let $X$ be a Banach space such that $\lim _{\epsilon \rightarrow 0} \delta \delta(\epsilon) / \epsilon<1$. Then $X$ has the weak Banach-Saks property.

Proof. By assumption, there exist $c \in(0,1)$ and $\eta>0$ such that if $0<\epsilon<\eta$, then $\delta \delta(\epsilon)<c \epsilon$. In light of Corollary 2.6, we can therefore find $E \in \mathscr{B}$ so that $b(\epsilon ; x)<c \epsilon$ for every $x \in S_{E}$.

Assume that $X$ does not have the weak Banach-Saks property. Fix $0<\epsilon<$ $\min \{\eta, 1\}$ and put $\gamma=(1-c) \epsilon / 6$. Then there exists a weakly null sequence $\left(y_{n}\right)$ in $X$ such that

$$
(1-\gamma)(|\alpha|+|\beta|) \leq\left\|\alpha y_{m}+\beta y_{n}\right\| \leq(1+\gamma)(|\alpha|+|\beta|)
$$

for all $m<n$ and $\alpha, \beta \in \mathbb{R}$ (see [3]).

Similarly, as in the proof of Theorem 2.5 we can choose $m$ and $y \in S_{E}$ for which $\left\|y_{m}-y\right\|<3 \gamma$. Clearly

$$
\inf _{n>m}\left\|y_{m}+\epsilon y_{n}\right\| \leq \inf _{n>m}\left\|y-\epsilon y_{n}\right\|+3 \gamma \leq b(\epsilon ; y)+3 \gamma<1+c \epsilon+3 \gamma
$$


But

$$
\inf _{n>m}\left\|y_{m}+\epsilon y_{n}\right\| \geq(1-\gamma)(1+\epsilon)
$$

which leads to a contradiction.

The weakly convergent sequence coefficient (WCS for short) was introduced in [4]. Here, we use a formula from [1, Lemma VI.3.8]. Let $X$ be a space without the Schur property. Then $\operatorname{WCS}(X)$ equals the infimum of all double limits $\lim _{n \neq m}\left\|x_{n}-x_{m}\right\|$ where $\left(x_{n}\right) \in \mathcal{N}$ is a sequence for which the above limit exists.

Proposition 2.14. Let $X$ be a Banach space without the Schur property. Then

$$
\operatorname{WCS}(X) \geq 1+\beta d(1)
$$

Proof. Given $\gamma>0$, we find $E \in \mathscr{R}$ such that $d(1 ; x)>\beta d(1)-\gamma$ for every $x \in$ $S_{E}$. There is a sequence $\left(y_{n}\right) \in \mathcal{N}$ for which $\operatorname{WCS}(X)+\gamma>\lim _{n \neq m}\left\|y_{n}-y_{m}\right\|$. We can clearly assume that $\operatorname{WCS}(X)+\gamma>\left\|y_{n}-y_{m}\right\|$ for all $m, n$.

Similarly as in the proof of Theorem 2.5 we can choose $m$ and $y \in S_{E}$ so that $\left\|y_{m}-y\right\|<\gamma$. Then

$$
\mathrm{WCS}(X)+\gamma \geq \sup _{n \in \mathbb{N}}\left\|y-y_{n}\right\|-\gamma \geq 1+d(1 ; y)-\gamma>1+\beta d(1)-2 \gamma .
$$

Since $\gamma>0$ is arbitrary, this gives the desired estimate.

In [1], many applications of the WCS coefficient to the fixed point theory can be found. Here we only mention that if $\operatorname{WCS}(X)>1$, then $X$ has weak normal structure. In consequence, $X$ has the weak fixed point property which means that each nonexpansive mapping of nonempty weakly compact set in $X$ has a fixed point (see [12]). We therefore obtain the following corollary of Proposition 2.14 and Corollary 2.6.

Corollary 2.15. Let $X$ be a Banach space. If $\beta \beta(1)>0$, then $X$ has the weak fixed point property.

\section{Acknowledgements}

The authors wish to express their gratitude to the referee for several helpful comments.

The second and third authors were partially supported by the State Committee for Scientific Research of Poland (KBN) grant no 2P03A02915. 


\section{References}

[1] J. M. Ayerbe Toledano, T. Domínguez Benavides, and G. López Acedo, Measures of Noncompactness in Metric Fixed Point Theory, Operator Theory: Advances and Applications, vol. 99, Birkhäuser Verlag, Basel, 1997. MR 99e:47070. Zbl 885.47021.

[2] J. Banaś, On moduli of smoothness of Banach spaces, Bull. Polish Acad. Sci. Math. 34 (1986), no. 5-6, 287-293. MR 88b:46026. Zbl 606.46010.

[3] B. Beauzamy, Banach-Saks properties and spreading models, Math. Scand. 44 (1979), no. 2, 357-384. MR 81a:46018. Zbl 427.46007.

[4] W. L. Bynum, Normal structure coefficients for Banach spaces, Pacific J. Math. 86 (1980), no. 2, 427-436. MR 81m:46030. Zbl 442.46018.

[5] M. M. Day, Uniform convexity in factor and conjugate spaces, Ann. of Math. (2) 45 (1944), 375-385. MR 6,69c. Zbl 063.01058.

[6] J. Diestel, Sequences and Series in Banach Spaces, Graduate Texts in Mathematics, vol. 92, Springer-Verlag, New York, 1984. MR 85i:46020. Zbl 542.46007.

[7] T. Figiel, On the moduli of convexity and smoothness, Studia Math. 56 (1976), no. 2, 121-155. MR 54\#13535. Zbl 344.46052.

[8] G. Godefroy, N. J. Kalton, and G. Lancien, Szlenk indices and uniform homeomorphisms, preprint.

[9] K. Goebel, Convexity of balls and fixed-point theorems for mappings with nonexpansive square, Compositio Math. 22 (1970), 269-274. MR 42\#8355. Zbl 202.12802.

[10] K. Goebel and T. Sẹkowski, The modulus of noncompact convexity, Ann. Univ. Mariae Curie-Skłodowska Sect. A 38 (1984), 41-48. MR 87j:46031. Zbl 607.46011.

[11] R. Huff, Banach spaces which are nearly uniformly convex, Rocky Mountain J. Math. 10 (1980), no. 4, 743-749. MR 82b:46016. Zbl 505.46011.

[12] W. A. Kirk, A fixed point theorem for mappings which do not increase distances, Amer. Math. Monthly 72 (1965), 1004-1006. MR 32\#6436. Zbl 141.32402.

[13] P.-K. Lin, $k$-uniform rotundity is equivalent to $k$-uniform convexity, J. Math. Anal. Appl. 132 (1988), no. 2, 349-355. MR 89i:46018. Zbl 649.46014.

[14] J. Lindenstrauss, On the modulus of smoothness and divergent series in Banach spaces, Michigan Math. J. 10 (1963), 241-252. MR 29\#6316. Zbl 115.10001.

[15] E. Maluta and S. Prus, Banach spaces which are dual to k-uniformly convex spaces, J. Math. Anal. Appl. 209 (1997), no. 2, 479-491. MR 98j:46014. Zbl 885.46011.

[16] V. D. Milman, Geometric theory of Banach spaces. II: Geometry of the unit sphere, Russian Math. Surveys 26 (1971), no. 6, 79-163, [translated from Uspehi Mat. Nauk 26 (1971) 73-149. MR 54:8240]. Zbl 238.46012.

[17] _ Duality of certain geometric characteristics of a Banach space, Teor. Funkciǐ Funkcional. Anal. i Priložen. (1973), no. 18, 120-137 (Russian). MR 48\#12004.

[18] V. D. Milman and A. Perelson, Infinite-dimensional geometric moduli and typecotype theory, Geometric Aspects of Banach Spaces, Lecture Note Series, vol. 140, Cambridge Univ. Press, Cambridge, 1989, pp. 11-38. MR 91d:46009. Zbl 827.46006.

[19] S. Prus, Nearly uniformly smooth Banach spaces, Boll. Un. Mat. Ital. B (7) 3 (1989), no. 3, 507-521. MR 91a:46017. Zbl 698.46014. 
[20] _ Banach spaces and operators which are nearly uniformly convex, Dissertationes Math. (Rozprawy Mat.) 363 (1997), 1-46. MR 98h:46015. Zbl 886.46017.

[21] I. Singer, Bases in Banach Spaces. II, Editura Academiei Republicii Socialiste România, Bucharest, 1981. MR 82k:46024. Zbl 467.46020.

[22] F. Sullivan, A generalization of uniformly rotund Banach spaces, Canad. J. Math. 31 (1979), no. 3, 628-636. MR 80h:46023. Zbl 422.46011.

Elisabetta Maluta: Dipartimento di Matematica, Politecnico di Milano, Via BonaRdi 9, 20133 Milano, Italy

E-mail address: elimal@mate.polimi.it

StanisŁaw Prus: Department of Mathematics, M. Curie-SkŁodowska UniverSITY, 20-031 Lublin, PolAND

E-mail address: bsprus@golem.umcs.lublin.pl

Mariusz Szczepanik: Department of Mathematics, M. Curie-SKŁodowsKa UniVersity, 20-031 Lublin, Poland

E-mail address: szczepan@golem.umcs.lublin.pl 


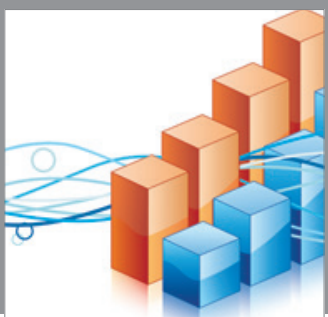

Advances in

Operations Research

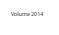

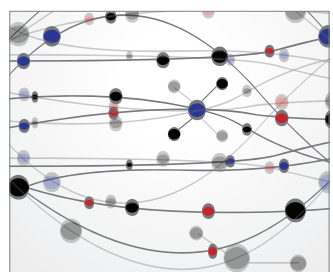

\section{The Scientific} World Journal
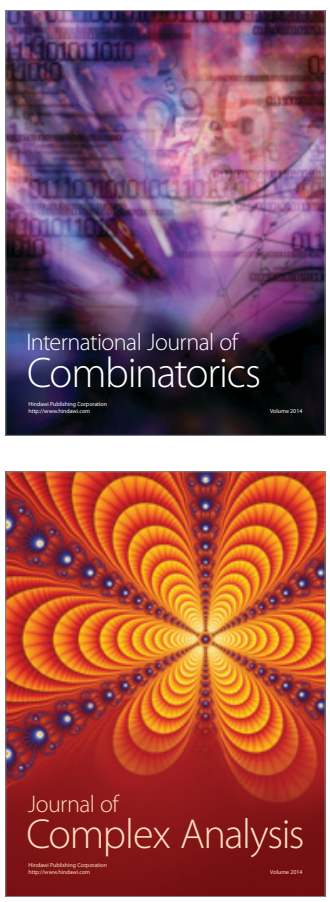

International Journal of

Mathematics and

Mathematical

Sciences
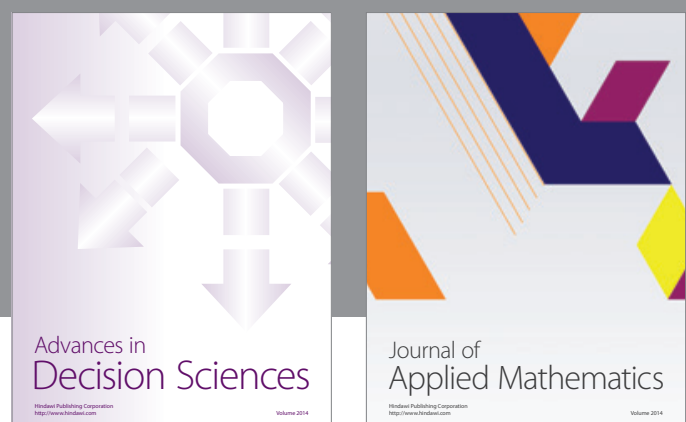

Journal of

Applied Mathematics
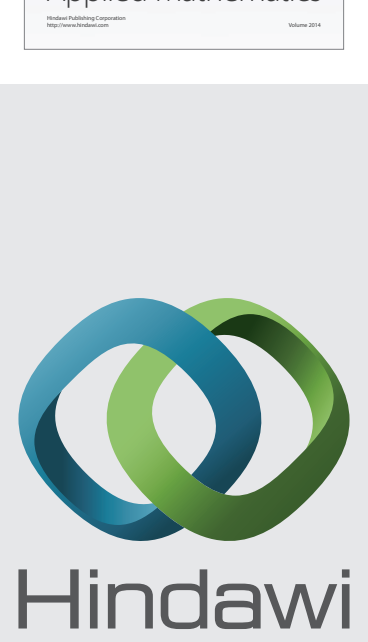

Submit your manuscripts at http://www.hindawi.com
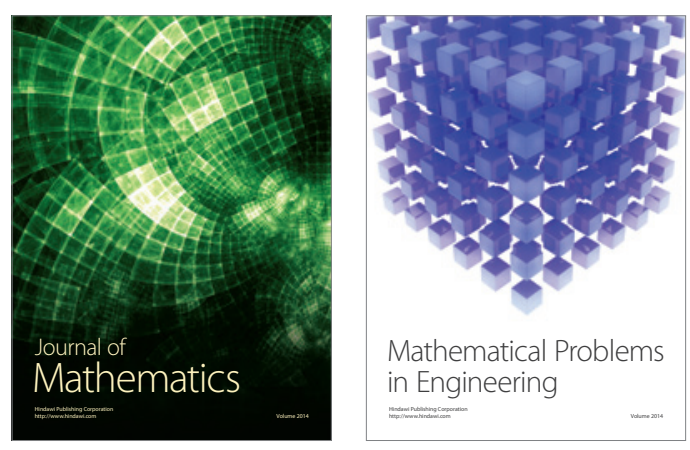

Mathematical Problems in Engineering
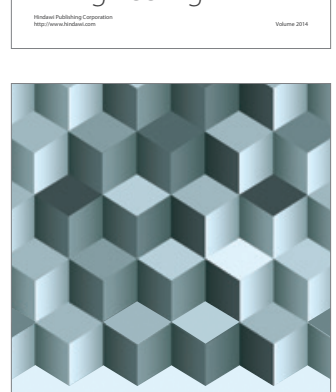

Journal of

Function Spaces
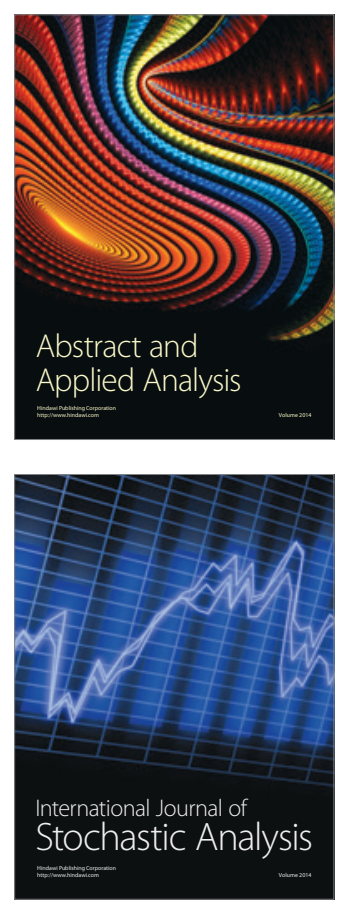

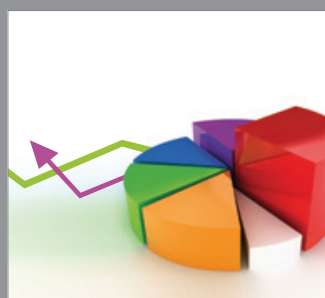

ournal of

Probability and Statistics

Promensencen
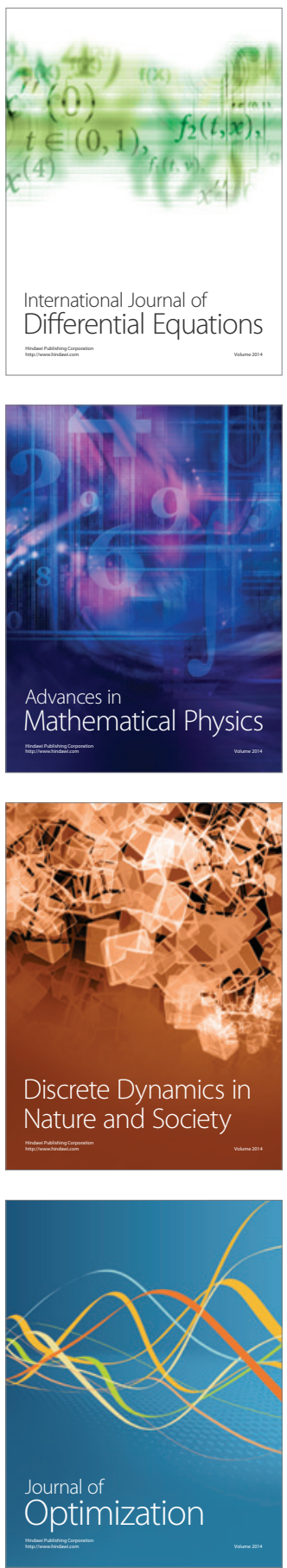